\title{
Population genomics and the evolution of virulence in the fungal pathogen Cryptococcus neoformans
}

\author{
Christopher A. Desjardins, ${ }^{1}$ Charles Giamberardino, ${ }^{2}$ Sean M. Sykes, ${ }^{1}$ Chen-Hsin $\mathrm{Yu}^{2}$ \\ Jennifer L. Tenor, ${ }^{2}$ Yuan Chen, ${ }^{2}$ Timothy Yang, ${ }^{2}$ Alexander M. Jones, ${ }^{2}$ Sheng Sun, ${ }^{3}$ \\ Miriam R. Haverkamp, ${ }^{4}$ Joseph Heitman, ${ }^{3}$ Anastasia P. Litvintseva, ${ }^{5}$ John R. Perfect, ${ }^{2}$ \\ and Christina A. Cuomo ${ }^{1}$ \\ ${ }^{1}$ Broad Institute of MIT and Harvard, Cambridge, Massachusetts 02142, USA; ${ }^{2}$ Division of Infectious Diseases, Department of \\ Medicine, Duke University Medical Center, Durham, North Carolina 27710, USA; ${ }^{3}$ Department of Molecular Genetics and \\ Microbiology, Duke University Medical Center, Durham, North Carolina 27710, USA; ${ }^{4}$ Perelman School of Medicine, University of \\ Pennsylvania, Philadelphia, Pennsylvania 19104, USA; ${ }^{5}$ Mycotic Diseases Branch, National Center for Emerging and Zoonotic \\ Infectious Diseases, Centers for Disease Control and Prevention, Atlanta, Georgia 30329, USA
}

\begin{abstract}
Cryptococcus neoformans is an opportunistic fungal pathogen that causes approximately 625,000 deaths per year from nervous system infections. Here, we leveraged a unique, genetically diverse population of $C$. neoformans from sub-Saharan Africa, commonly isolated from mopane trees, to determine how selective pressures in the environment coincidentally adapted C. neoformans for human virulence. Genome sequencing and phylogenetic analysis of 387 isolates, representing the global VNI and African VNB lineages, highlighted a deep, nonrecombining split in VNB (herein, VNBI and VNBII). VNBII was enriched for clinical samples relative to $\mathrm{VNBI}$, while phenotypic profiling of 183 isolates demonstrated that VNBI isolates were significantly more resistant to oxidative stress and more heavily melanized than VNBII isolates. Lack of melanization in both lineages was associated with loss-of-function mutations in the BZP4 transcription factor. A genome-wide association study across all VNB isolates revealed sequence differences between clinical and environmental isolates in virulence factors and stress response genes. Inositol transporters and catabolism genes, which process sugars present in plants and the human nervous system, were identified as targets of selection in all three lineages. Further phylogenetic and population genomic analyses revealed extensive loss of genetic diversity in VNBI, suggestive of a history of population bottlenecks, along with unique evolutionary trajectories for mating type loci. These data highlight the complex evolutionary interplay between adaptation to natural environments and opportunistic infections, and that selection on specific pathways may predispose isolates to human virulence.
\end{abstract}

[Supplemental material is available for this article.]

Cryptococcus neoformans is an opportunistic fungal pathogen of enormous clinical importance. An estimated one million new Cryptococcus infections occur each year resulting in approximately 625,000 deaths (Park et al. 2009). In Africa, cryptococcosis is the third most common cause of death in patients with HIV/AIDS, and mortality from cryptococcal meningitis exceeds the death rate from tuberculosis (Park et al. 2009). Of the two varieties recognized within the C. neoformans species complex, >95\% of cryptococcosis cases are caused by C. neoformans var. grubii (serotype A lineage) (Chayakulkeeree and Perfect 2006).

Genetic analyses have subdivided $C$. neoformans var. grubii into three distinct lineages. The VNI and VNII lineages are highly clonal and globally distributed, whereas the third lineage, $\mathrm{VNB}$, is genetically diverse yet largely restricted to sub-Saharan Africa (Litvintseva et al. 2006). Environmental reservoirs also differ between these groups; VNB is associated with collection from tree hollows and soil at the base of trees, particularly the mopane tree (Colophospermum mopane), which predominates in the African sa-

Corresponding authors: cuomo@broadinstitute.org, john.perfect@ duke.edu

Article published online before print. Article, supplemental material, and publication date are at http://www.genome.org/cgi/doi/10.1101/gr.218727.116. Freely available online through the Genome Research Open Access option. vannah (Litvintseva et al. 2011). VNI is associated with both trees and pigeon excreta (Litvintseva et al. 2011), whereas the environmental reservoir for VNII is not well defined, as the vast majority of known VNII isolates have a clinical origin.

The three lineages also differ in the prevalence of each of the two mating types; however, the extent to which this impacts recombination is not well understood. In Cryptococcus, the two mating types, $\mathbf{a}$ and $\alpha$, are encoded by a $\sim 100-\mathrm{kb}$ mating type locus (MAT) (Lengeler et al. 2002). Sexual reproduction and meiotic recombination can result both from bisexual mating of the opposite mating types as well as from unisexual mating between MAT $\alpha$ isolates (Lin et al. 2005). VNI and VNII are predominantly of a single mating type $(M A T \alpha)$, whereas VNB contains a mix of both mating types (MAT $\alpha$ and MATa); the presence of both mating types in VNB has led to the suggestion of more extensive recombination in this group compared to VNI and VNII (Litvintseva et al. 2003, 2006). Previous genetic analyses of $C$. neoformans suggested recombination in both single and multiple mating type populations (Litvintseva et al. 2003, 2006; Hiremath et al. 2008), although these studies were based on amplified fragment length

(C) 2017 Desjardins et al. This article, published in Genome Research, is available under a Creative Commons License (Attribution 4.0 International), as described at http://creativecommons.org/licenses/by/4.0/. 
polymorphism (AFLP) or multilocus sequence typing (MLST) data and therefore could only provide limited resolution.

C. neoformans is an environmental opportunistic pathogen, incapable of human-to-human transmission or dissemination back to the environment from infected hosts. Therefore direct selection of traits to confer virulence or transmission advantages in humans cannot occur. Rather, traits with increased virulence in humans are the result of coincidental selection (Brown et al. 2012), resulting from interactions of $C$. neoformans with other eukaryotes such as predatory amoeba and nematodes in natural environments (Steenbergen et al. 2001). Isolates collected from the environment have been shown to be less virulent in animal models than those recovered from clinical sources (Fromtling et al. 1989; Litvintseva and Mitchell 2009), suggesting that there are advantageous genotypes for growth in a human host. Understanding the selective forces operating on C. neoformans in nature is critical to understanding genetic diversity as it relates to virulence in humans.

Three primary virulence factors for $C$. neoformans include a polysaccharide capsule that inhibits phagocytosis, melanin that protects from environmental stresses, and the ability to grow at human body temperature, $37^{\circ} \mathrm{C}$ (Bulmer et al. 1967; KwonChung and Rhodes 1986). Numerous additional virulence factors have been identified in $C$. neoformans, such as phospolipase $\mathrm{B}$ (Cox et al. 2001), urease (Cox et al. 2000), and a number of signaling cascades (Kozubowski et al. 2009). Although the importance of these genes has been confirmed by gene deletion, natural genetic variation encoded within these and other factors could also affect virulence in a quantitative fashion, as genetically similar isolates have been identified that have differential ability to cause disease in animals (Litvintseva and Mitchell 2009).

Previous genomic work has provided reference assemblies of C. neoformans (Loftus et al. 2005; Janbon et al. 2014) and the sister species C. gattii (D'Souza et al. 2011; Farrer et al. 2015). Here, we leverage the reference assembly of $C$. neoformans strain H99 to conduct large-scale sequencing and variant calling of 387 isolates of $C$. neoformans var. grubii, covering the phylogenetic, geographic, and ecological breadth of the species. We trace the complex evolutionary history of the group, using phylogenetic and population genomic analyses, and dissect the genetic differences between clinical and environmental isolates through tests for genome-wide association and selection. For the latter analyses, we focus on VNB, as the high genetic diversity and suggested frequent recombination in the lineage, along with ease of isolation from both clinical and environmental sources, make it particularly suited to these tests. Furthermore, we conduct a high-throughput phenotypic screen of $183 \mathrm{VNB}$ isolates to explore how genetic differences relate to responses to environmental stresses. These analyses provide insight into how genetic variation relates to clinical prevalence and how selection in the environment has adapted C. neoformans for infection of human hosts.

\section{Results}

\section{Phylogenetic analysis reveals deep, nonrecombining split} in VNB lineage

To better understand the population structure and genetic basis of virulence across $C$. neoformans var. grubii lineages, we sequenced and identified variants for 387 isolates, including 185 from VNI, 186 from VNB, and 16 from VNII (Methods; Supplemental Table S1). A phylogeny estimated from approximately 1 million variable positions showed clear separation of the three monophyletic lineages VNI, VNII, and VNB (Fig. 1). However, inspection of the phylogeny also revealed a strongly supported (100\% bootstrap support), deep split within the VNB lineage that matched a division seen in previous analyses of AFLP data (Litvintseva et al. 2003), MLST data (Litvintseva et al. 2006; Chen et al. 2015), four nuclear loci (Ngamskulrungroj et al. 2009), and 23 whole-genome sequences (Vanhove et al. 2017). This split was further supported by analysis of population ancestry using STRUCTURE $(k=4)$ and of sequence similarity using principal components analysis (PCA), both of which produced four distinct groups representing VNI, VNII, and two VNB lineages with little to no intermixing (Fig. 2A,B). We therefore propose that VNB be subdivided into two distinct lineages, VNBI and VNBII, following the nomenclature of Chen et al. (2015). VNBI encompassed 122 isolates from this study, including previously studied MATa isolates Bt63 and Bt204; VNBII included 64 isolates from this study, including previously studied MATa isolates Bt65 and Bt206 (Litvintseva et al. 2003, 2006; Morrow et al. 2012).

To determine if these groups represented reproductively isolated, nonrecombining lineages, we calculated decay of linkage disequilibrium (LD) for VNI, VNBI, VNBII, all VNB, and VNB combined with VNI. We excluded VNII from the calculations due to small sample size. Neither the combined set of VNB and VNI, nor VNB alone, demonstrated evidence of 50\% LD decay within the 250-kb range of the analysis (Fig. 2C), suggesting limited recombination between members of these populations. However, when split into VNI, VNBI, and VNBII, all lineages demonstrated $50 \%$ LD decay in 5-7.5 kb (Table 1; Fig. 2C). This level of recombination is similar to that previously reported for wild populations of Saccharomyces cerevisiae and S. paradoxus (Liti et al. 2009).

In the phylogenetic analysis, VNI was further subdivided into three distinct clades, two of which were globally distributed (VNIa and VNIb) and one of which was restricted to sub-Saharan Africa (VNIc) (Supplemental Fig. S1). These three clades were highly supported (100\% bootstrap support) in this phylogeny and were consistent with clades identified in a prior MLST analysis (Litvintseva et al. 2006). In contrast, a more recent MLST study identified two major VNI subdivisions, although with low bootstrap support (Ferreira-Paim et al. 2017); these subdivisions both fell within VNIa in our analysis (Methods). The VNIa clade contains the highest diversity ( $\pi=0.00178)$ compared to VNIc $(0.00125)$ and VNIb (0.00084). STRUCTURE analysis and PCA showed the three clades to be largely distinct (Supplemental Fig. S1B), with a small subset of isolates showing evidence of mixed ancestry of all three clades. However, faster LD decay was observed in the full VNI set of isolates compared to that of the individual subclades (Supplemental Fig. S1C), suggesting there is active recombination between the VNI subclades and that VNI should be considered a single lineage.

To determine whether there was some level of genetic exchange between the groups below the level of detection of genome-wide LD and STRUCTURE analyses, we repeated the STRUCTURE analysis at 500-kb intervals and identified 46 isolates with evidence of recent introgressions of at least $50 \mathrm{~kb}$ from different VN groups (Fig. 3; Methods). These introgressions were composed of segments ranging in size from 5 to $260 \mathrm{~kb}(0.02 \%-1.4 \%$ of the genome) and occurred in all pairwise combinations of VNI, VNBI, and VNBII as recipient and donor; VNII donors but not recipients were detected. Thirty-two VNBI isolates contained introgressions, which was significantly more than the $14 \mathrm{VNI}$ isolates and five VNBII isolates that contained introgressions $(P<$

\section{Genome Research}

www.genome.org 


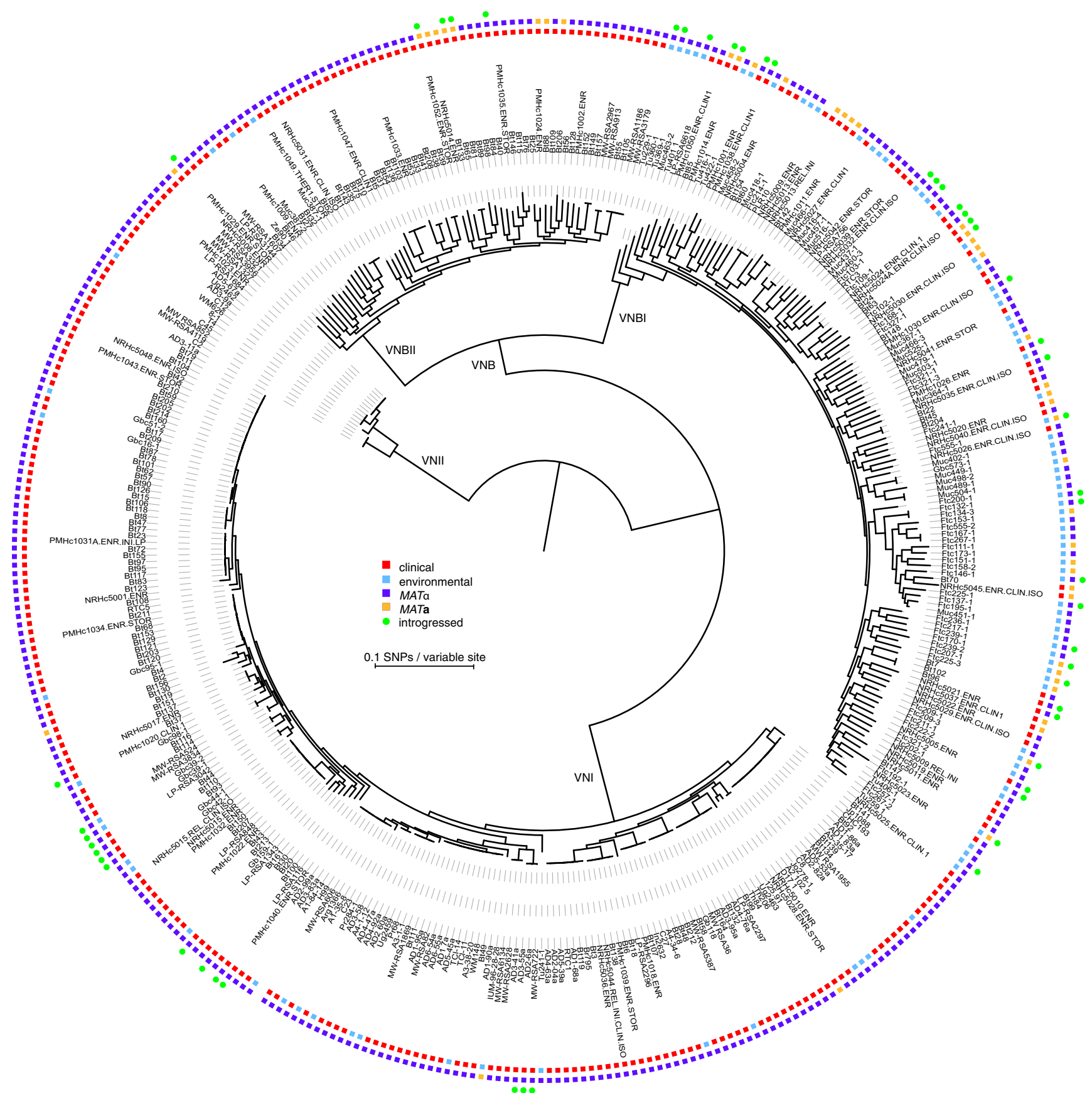

Figure 1. Phylogenomic analysis reveals a deep split within the VNB lineage. We propose that VNB be divided into two lineages: VNBI and VNBII. The phylogeny was estimated from 1,069,080 segregating sites using RAxML (Stamatakis 2014), and the tree was rooted with VNII as the outgroup. All lineages (VNI, VNII, VNBI, and VNBII) had 100\% bootstrap support. Isolation source (clinical versus environmental), mating type (MAT $\alpha$ and MATa), and presence of $\geq 50 \mathrm{~kb}$ of introgressions from other lineages are also shown. VNBI samples were enriched for environmental isolation sources relative to VNBII $(P<0.0001$, Fisher's exact test).

$1.32 \times 10^{-5}$ and $P<0.0033$, respectively, Fisher's exact test). Isolates with introgressions included both mating types and were dispersed across the VNB phylogeny, but within VNI only occurred within the African-specific subclade (Fig. 1). Overall, introgressed regions were not functionally enriched for any specific gene functions; however, the most common introgression in VNBI (present in 14 clinical and environmental isolates, from VNI) encompasses $40 \mathrm{~kb}$ and encodes a glycolipid mannosyltransferase involved in capsule synthesis (CNAG_00926) (O'Meara and Alspaugh 2012) and secretome component carboxypeptidase D (Geddes et al. 2015). Conversely, the most common introgression in VNI (present in eight clinical and environmental isolates, from VNBI) encompasses $60 \mathrm{~kb}$ and contains virulence factor VCX1 (Kmetzsch et al. 2010) and azole drug target ERG11. This suggests a low level of mixing of genomic regions responsible for clinically relevant phenotypes may be occurring between lineages in Africa. 
A

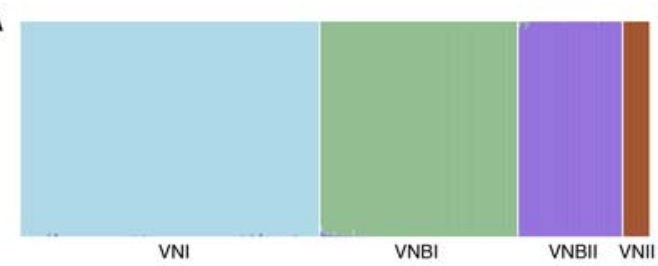

B

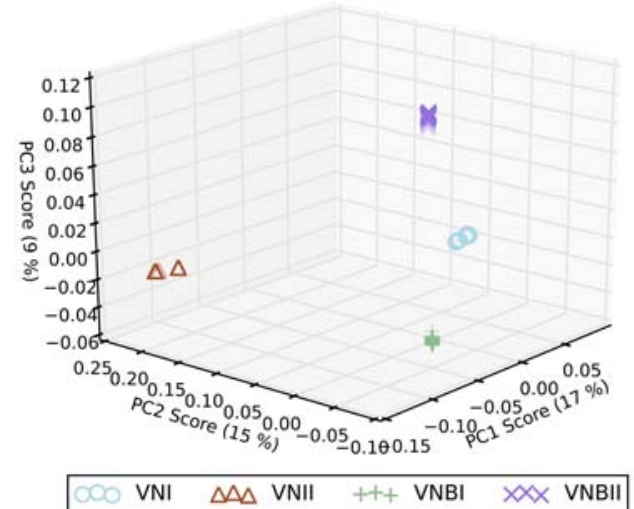

C

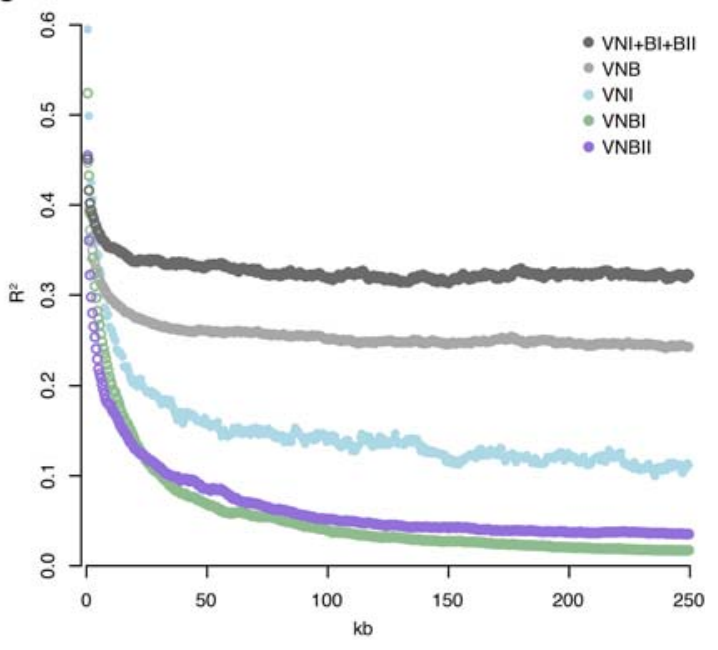

Figure 2. C. neoformans var. grubii is separated into four distinct nonrecombining lineages: VNI, VNII, VNBI, and VNBII. ( $A$ ) Results of STRUCTURE analysis $(k=4)$ shows separation of all four lineages. (B) Top three principal components from PCA. The third principal component clearly separates VNBI from VNBII. (C) Decay of linkage disequilibrium (LD) shows similar rates of recombination within groups and lack of recombination between groups. $L D\left(R^{2}\right)$ was calculated for all pairs of SNPs separated by $0-250 \mathrm{~kb}$ and then averaged for every $500 \mathrm{bp}$. LD values for each window were then calculated by averaging over all pairwise calculations in the window. The chromosome with the mating type locus was excluded from the calculations.

\section{VNBII isolates are enriched for clinical isolation source relative to VNBI}

Although the VNB lineage was previously associated with a high frequency of environmental samples and the a mating type, we found differences between the relative frequencies of these properties between VNBI and VNBII. Whereas VNBI contained large numbers of both clinical and environmental samples (52 versus 70, respectively), VNBII contained almost exclusively clinical isolates (60 versus three, respectively), which was a significant difference $(P<0.0001$, Fisher's exact test). Environmental isolates in both VNBI and VNBII were scattered across the phylogenetic tree

Table 1. Genomic and population characteristics of global sampling of the four lineages of $C$. neoformans var. grubii

\begin{tabular}{lllll}
\hline Statistic & \multicolumn{1}{c}{ VNI } & VNBI & VNBII & VNII \\
\hline Sample size & 185 & 122 & 64 & 16 \\
LD50 (kb) & 7.5 & 6 & 5 & ND \\
$\pi$ & 0.00209 & 0.00360 & 0.00358 & ND \\
Reduced TD (\%) & 1.8 & 12 & 2.4 & ND \\
Metadata & & & & \\
$\quad$ Clinical (\#) & 160 & 51 & 61 & 15 \\
Environmental (\#) & 25 & 71 & 3 & 1 \\
MAT $(\#)$ & 182 & 88 & 55 & 16 \\
MATa (\#) & 3 & 33 & 9 & 0 \\
Clinical/environmental (\%) & NA & $42 / 58$ & $95 / 6$ & $94 / 6$ \\
MATa/ $\alpha$ (\%) & NA & $27 / 72$ & $14 / 86$ & NA \\
\hline
\end{tabular}

Listed statistics include sample size, physical distance needed for linkage disequilibrium to decay $50 \%$ (LD50), diversity $(\pi)$, percentage of the genome with reduced Tajima's $D$ (TD), and number and percentage of isolates isolated from clinical and environment sources, and of each mating type (MAT $\alpha$ or MATa). Given the limited sampling of VNII, many statistics were not determined (ND) or not applicable (NA). and showed no evidence of clustering, although all three environmental VNBII isolates were relatively basal in the lineage (Fig. 1). SNP calling and phylogenetic analysis of 23 recently published Zambian isolates (Vanhove et al. 2017) showed a similar bias in environmental and clinical isolates in the two lineages; the Zambian mixed environmental and clinical VNB-A clade matched our VNBII lineage, whereas the VNB-B clade, which was entirely environmental samples, matched our VNBI lineage (Supplemental Fig. S2). Furthermore, VNBI had a greater proportion of MATa isolates than VNBII (33/122 versus 9/64, respectively), although the difference was not significant.

Analysis of annotated whole-genome assemblies of representatives of the four lineages revealed very few gene gains or losses specific to VNBI and VNBII (Supplemental Table S2). Most gains and losses were hypothetical proteins, with the exceptions of a gain of an $\alpha-\beta$ hydrolase and loss of a phosphopyruvate hydratase and an alginate lyase in VNBI, and a gain of an L-iditol 2-dehydrogenase in VNBII. However, a large number of fixed polymorphisms were present between the two lineages, in addition to private unfixed alleles in each lineage and unfixed alleles shared between the two lineages (Supplemental Fig. S3A). Therefore small polymorphisms may underlie much of the phenotypic variation within and between these lineages.

To identify genomic features that might contribute to increased prevalence in clinical populations, we conducted a genome-wide association analysis (GWAS) of small polymorphisms and loss-of-function mutations across VNBI and VNBII. Correction for population stratification was included so that features that evolved at the base of VNBI would not overwhelm the analysis. The top 10 loci associated with the clinical phenotype included a number of known virulence factors and genes involved in oxidative stress response (Table 2). The top scoring locus was the intergenic region downstream from CNAG_07989 $\left(P<3.0 \times 10^{-7}\right.$,

\section{Genome Research}

www.genome.org 


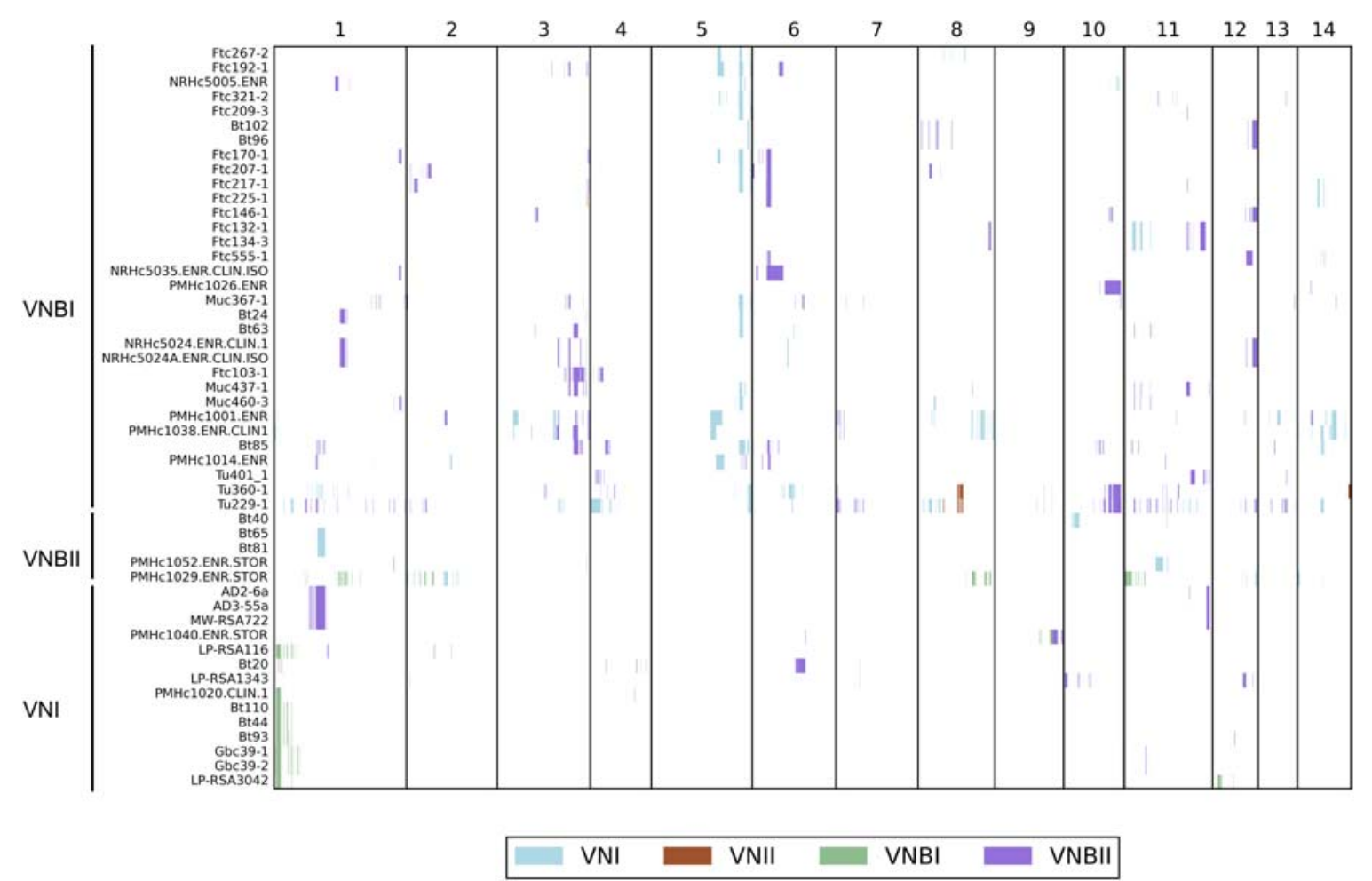

Figure 3. Small introgressions between VNI, VNII, VNBI, and VNBII are dispersed throughout the genome and phylogeny. Introgressions were detected by running STRUCTURE $(k=4)$ on $500-\mathrm{kb}$ blocks, excluding the mating type locus, and the group ancestry of each $5 \mathrm{~kb}$ within each block was identified. Recipient genomes are shown on the $y$-axis, and genomic position is shown on the $x$-axis. White indicates non-introgressed regions, and colored blocks indicate introgressed regions. The color key shows the color that corresponds to each donor group.

score test), which encodes a velvet family protein, and a deletion of that gene prevents filamentation (Chacko et al. 2015). Also identified were the intergenic region downstream from LMP1, a known virulence factor identified through laboratory passage of H99 (Janbon et al. 2014), CCK2, which encodes a casein kinase related to CCK1 (essential for cell integrity and virulence (Wang et al. 2011)), and three genes, PDX3, FOX21, and FHB1, previously noted to respond to oxidative and nitrosative stress (de Jesús-Berríos et al. 2003; Upadhya et al. 2013).

We also examined geographical associations of the VNBI and VNBII lineages. VNBII isolates were isolated almost exclusively from patients treated at the Princess Marina Hospital in southern Botswana, whereas VNBI isolates were common in all four heavily sampled locations, including patients from both hospitals and the environment in south, northeast, and north-central Botswana (Supplemental Table S3). Further evaluation of the VNBI population revealed little evidence for genetic structure separating or connecting specific locations (Supplemental Table S4). We also examined the combined phylogeny (Supplemental Fig. S2) of the Botswana isolates with the previously sequenced Zambian isolates (Vanhove et al. 2017) to determine whether these patterns extended outside of Botswana. Surprisingly, both VNBI and VNBII Zambian isolates largely clustered near the base of each lineage, suggesting some genetic separation between Botswanan and Zambian VNB lineages and that some geographic isolation may be occurring at the scale of country. The Zambian VNI isolates, on the other hand, were dispersed throughout our global VNIa and VNIb clades, although not the African VNIc clade, suggesting greater gene flow between VNI populations in the region.
VNBI and VNBII differ in their ability to melanize and respond to oxidative stress

To determine if the distinct genetic backgrounds of VNBI and VNBII, or variants identified in the association analysis, correlated with any relevant phenotypes, we assayed 183 of the 186 sequenced VNBI and VNBII isolates for growth under a variety of stress conditions, along with a set of control isolates including the VNI reference strain H99. We specifically tested response to oxidative stressors $\mathrm{H}_{2} \mathrm{O}_{2}$ and paraquat, melanization capacity with $\mathrm{L}$ DOPA, and resistance to the antifungal drug fluconazole (for results, see Supplemental Table S5). We did not identify any isolates with mutations in stress response genes PDX3, FOX21, or FHB1 with extreme response phenotypes. However, we did identify significant phenotypic differences between VNBI and VNBII isolates and between clinical and environmental isolates. To control for the interaction of lineage and isolation source, we analyzed only clinical isolates for lineage comparisons and only VNBI isolates for isolation source comparisons. We also excluded all isolates that were completely unable to melanize in the comparison of melanization between environmental and clinical isolates to prevent any effect of sampling bias (Methods).

VNBI clinical isolates were significantly more melanized than VNBII clinical isolates $(P<0.00017$, Mann-Whitney $U$ test) (Fig. $4 \mathrm{~A})$ and significantly more resistant to both $\mathrm{H}_{2} \mathrm{O}_{2}(P<0.0049$, Mann-Whitney $U$ test) (Supplemental Fig. S4A) and paraquat ( $P$ $<0.042$, Mann-Whitney $U$ test) (Supplemental Fig. S4B), but not fluconazole $(P<0.78$, Mann-Whitney $U$ test) (Supplemental Fig. $\mathrm{S} 4 \mathrm{C}$ ). Within VNBI, environmental isolates were more heavily 
Table 2. GWAS analysis reveals loci associated with the clinical phenotype in VNBI and VNBII

\begin{tabular}{|c|c|c|c|c|}
\hline$P$-value & Hit type & Locus & Gene(s) & References \\
\hline $2.02 \times 10^{-7}$ & LOF & CNAG_01348 & Cyanate hydratase & \\
\hline $3.00 \times 10^{-7}$ & Rare & Intergenic: CNAG_07989-CNAG_07661 & $\begin{array}{l}\text { Velvet family protein, deletion prevents filamentation; } \\
\text { hypothetical protein }\end{array}$ & Chacko et al. (2015) \\
\hline $3.22 \times 10^{-7}$ & Common & Intergenic: CNAG_07972-CNAG_06879 & Chromosome 3 centromeric region & \\
\hline $1.19 \times 10^{-6}$ & Rare & CNAG_05440 & $\begin{array}{l}\text { Pyridoxamine } 5 \text { '-phosphate oxidase PDX3, down- } \\
\text { regulated under oxidative stress }\end{array}$ & Upadhya et al. (2013) \\
\hline $2.44 \times 10^{-6}$ & Rare & CNAG_03374 & DNA replication complex GINS protein PSF1 & \\
\hline $2.91 \times 10^{-6}$ & Common & Intergenic: CNAG_06765-CNAG_06764 & $\begin{array}{l}\text { Low mating performance } L M P 1 \text {, deletion is avirulent; } \\
\text { short chain dehydrogenase } F O X 21 \text {, down-regulated } \\
\text { under oxidative stress }\end{array}$ & $\begin{array}{l}\text { Janbon et al. (2014); } \\
\text { Upadhya et al. } \\
\text { (2013) }\end{array}$ \\
\hline $3.99 \times 10^{-6}$ & Common & CNAG_06921 & Hypothetical protein & \\
\hline $5.07 \times 10^{-6}$ & Common & CNAG_05037 & Hypothetical protein & \\
\hline $5.62 \times 10^{-6}$ & Rare & CNAG_07427 & $\begin{array}{l}\text { Casein kinase CCK2, related to } C C K 1 \text { which is essential } \\
\text { for virulence }\end{array}$ & Wang et al. (2011) \\
\hline $6.00 \times 10^{-6}$ & Rare & CNAG_01464 & Flavohemoglobin $F H B 1$, nitrosative stress response & $\begin{array}{l}\text { de Jesús-Berríos et al. } \\
\text { (2003) }\end{array}$ \\
\hline
\end{tabular}

Two GWAS analyses were conducted. In the first, variants $<5 \%$ frequency were combined by gene or intergenic region (rare), whereas variants $>5 \%$ frequency were treated independently (common). In the second analysis, loss-of-function mutations were identified and combined by gene (LOF). Both analyses were conducted using GEMMA corrected for population stratification with a relatedness matrix (Zhou and Stephens 2012). The 10 most significant loci according to the score test across both analyses are shown.

melanized $(P<0.00047$, Mann-Whitney $U$ test) than clinical isolates and more resistant to both $\mathrm{H}_{2} \mathrm{O}_{2} \quad(P<0.00098$, MannWhitney $U$ test $)$ and paraquat $\left(P<4.1 \times 10^{-5}\right.$, Mann-Whitney $U$ test). The groups did not significantly differ in their resistance to fluconazole $(P<0.071$, Mann-Whitney $U$ test).

We then conducted a second GWAS analysis to identify genotypic variants associated with increased or decreased responses in the phenotypic assays (Supplemental Tables S6-S9). These analyses revealed that loss-of-function mutations in BZP4 (CNAG_03346) were highly associated with reduced melanization ability $\left(P<3.43 \times 10^{-9}\right.$, score test). Four clinical isolates, two from VNBI and two from VNBII, each had a different loss-of-function mutation in BZP4. Three of these isolates had a reduced melanization ability similar to that of a lac1 deletion mutant, the primary gene required for melanization (Salas et al. 1996), whereas the fourth isolate showed a slightly reduced melanization potential (Fig. 4A-C). BZP4 was previously associated with a reduced melanization capacity in a deletion screen of $C$. neoformans transcription factors (Jung et al. 2015). We then further screened genes previously categorized as having melanization defects in another deletion screen (Liu et al. 2008) and identified a second gene, $\mathrm{CHO} 2$ (CNAG_03139), at lower rank in the association analysis $(P<$ 0.0083 , score test). Loss-of-function of this gene is present in all VNBII isolates and could be related to the reduced melanization capability of VNBII. Although GWAS analysis did not produce any promising candidates for fluconazole resistance, copy number analysis revealed a duplication of drug target ERG11 in the four most resistant isolates (Bt65, Bt89, MW-RSA3179, and MWRSA2967).

\section{Population genomic analysis identifies extensive loss of diversity in VNBI}

To better understand genetic diversity at the lineage level, we calculated a number of population genetic statistics for VNI, VNBI, and VNBII, including $\pi$, Tajima's $D$, and $F_{\text {ST }}$ (Table 1 ). Genomewide calculations of the diversity metric $\pi$ revealed that VNBI and VNBII had nearly twice the genetic diversity of VNI (Table 1). However, when diversity was plotted along each chromosome, VNBI and VNBII appeared quite different. As shown in Figure 5A for Chromosome 5, the VNI group contained long tracts of low diversity, whereas VNBII contained predominantly high diversity regions, as expected. In contrast, VNBI showed a unique pattern with high diversity regions interspersed with long tracts of low diversity. These regions of low diversity correspond with tracts of low Tajima's $D$, which suggests an excess of rare alleles resulting from a population bottleneck or recent selective sweep. Confirming this, both VNI and VNBI had significantly more rare alleles across the entire genome ( $<2 \%$ frequency) than VNBII $(P<2.2 \times$ $10^{-16}$ for both comparisons, Fisher's exact test) (Supplemental Fig. S3B). To determine if this pattern appeared in all chromosomes, we plotted the distribution of Tajima's $D$ values for each chromosome individually (Fig. 5B). Although VNI and VNBII have unimodal distributions for most chromosomes, VNBI has a bimodal distribution of Tajima's $D$ across nearly all chromosomes, with the possible exception of Chromosome 6 . However, when we evaluated genes located in the regions of reduced Tajima's $D$, we found no significant functional enrichment in any of the lineages.

In most cases, when two lineages had regions of low diversity and Tajima's $D$, they also had high $F_{\mathrm{ST}}$. However, there was one notable exception to this on Chromosome 5, where a region of reduced diversity in both VNBI and VNBII included a region of high $F_{\mathrm{ST}}$ abruptly adjacent to a region of low $F_{\mathrm{ST}}$ (Supplemental Fig. S5). The low $F_{\mathrm{ST}}$ region includes CNAG_07442, which encodes a phosphatidylglycerol transfer protein that was the most abundant antigen recovered from an experimental mouse vaccine using strain cap59 (Specht et al. 2015), suggesting a similar antigen would be found in both lineages. The high $F_{\mathrm{ST}}$ region included CNAG_01058, a gene involved in capsule maintenance (Brown et al. 2014) and CNAG_01060, a potential substrate of the FBP1 gene required for proliferation in macrophages and dissemination (Liu and Xue 2014).

\section{Inositol and other sugar transporters are targets of selection in all VN lineages}

Because Tajima's $D$ can identify effects of both selection and demography (Tajima 1989), we used the composite likelihood ratio test (CLR) (Nielsen et al. 2005), which is specific to selection, in a genomic scan for regions under selection in each lineage

\section{Genome Research}

www.genome.org 


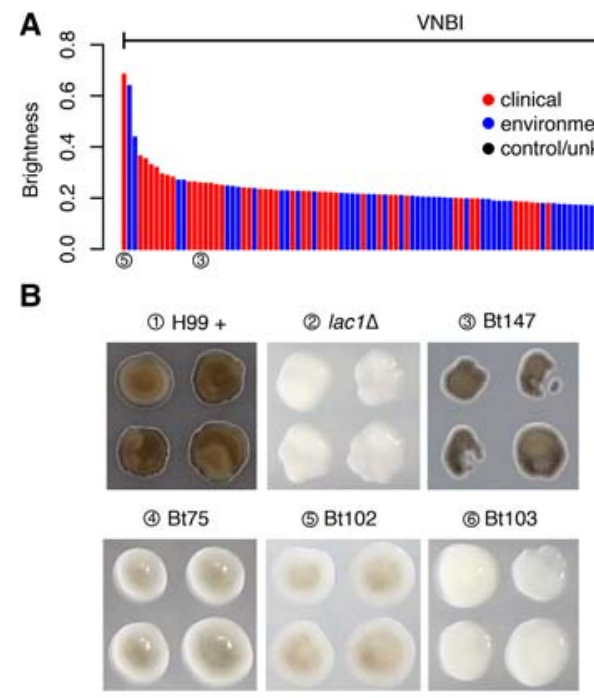

Figure 4. Phenotypic and GWAS analyses demonstrate that VNBI isolates have a greater ability to melanize than VNBII isolates and identify $B Z P 4$-deficient isolates as having reduced melanization capacity. $(A)$ Isolates were provided with L-DOPA, and colony brightness was assayed; isolates with the lowest brightness are the most melanized. Clinical isolates are shown in red, environmental isolates are shown in blue, control/unknown isolates are shown in black, and isolates described in $B$ are underscored with numbered circles. VNBI clinical isolates were significantly more melanized than VNBII clinical isolates $(P<0.00017)$, and VNBI environmental isolates were significantly more melanized than VNBI clinical isolates $(P<$ $0.00047)$. For the latter comparison, the least melanized (brightness $\geq 0.6$ ) samples were excluded to prevent an effect of sampling bias. (B) GWAS analysis identified loss-of-function mutations in BZP4 as being associated with a lack of melanization. The four isolates with BZP4 loss-of-function mutations are shown here in the L-DOPA assay, along with the positive control $\mathrm{H} 99$ and the negative control lac1 $1 \Delta$. (C) A strong correlation of melanization was present between replicates. Isolates shown in $B$ are indicated with numbered circles.

(Supplemental Fig. S6). Surprisingly, there was very little overlap between the genes in regions under positive selection and the genes in regions of reduced diversity in any of the lineages, suggesting that selective sweeps were not fixing genes under selection. To further evaluate genes under positive selection, we identified genes in regions with the top 5\% of CLR values for each of VNI, VNBI, and VNBII. A functional enrichment analysis of these genes revealed that major facilitator superfamily (MFS) transporters, and in particular the sugar transporter subset, were under selection in all lineages (Supplemental Table S10). Overlaying these genes onto a phylogeny of all sugar transporters in the reference strain H99 showed the selection was concentrated in transporters of inositol, xylose, maltose, $\alpha$-glucosides, lactose, and galactose (Supplemental Fig. S7). Six inositol transporters were found to be under selection in at least one lineage, including ITR1A (CNAG_04552), which was under selection in all three lineages (Table 3). Furthermore, genes involved in inositol catabolism, including four copies of inositol 2-dehydrogenase and one copy of inositol oxygenase, were also under selection, notably in VNI where all five genes were under selection. Other groups of genes under selection with enriched functions include monoxygenases in VNI and $\beta$-glucosidases in VNBII.

Subtelomeric regions of Cryptococcus have been previously highlighted as regions of high diversity heavily enriched for transporters (Chow et al. 2012). To determine if subtelomeric regions showed signatures of selection, we searched for enrichment of windows under selection in the outer $40 \mathrm{~kb}$ of each chromosome for VNI, VNBI, and VNBII. Chromosomes 1, 4, 5, and 14 showed subtelomeric enrichment of selection in all three examined lineages, and all chromosomes except 2 and 6 showed enrichment in at least one lineage (Supplemental Table S11). This suggests subtelomeric regions encode numerous targets of selection across the VNI, VNBI, and VNBII lineages. Enrichment analysis comparing genes under selection in the subtelomeric regions to the remainder of the genome showed the same patterns as the genome-wide selection analysis. Additionally, an enrichment of fungal transcription factors was identified (Supplemental Table S12), including FZC20, FZC41, SIP402, and FZC22, the last of which has been shown to have reduced virulence in mice when deleted (Jung et al. 2015).

\section{Mating type loci MAT $\alpha$ and MATa have distinct evolutionary trajectories}

The mating type locus also showed strong signatures of selection. To better understand the evolution of the MAT locus, we re-called SNPs against complete assemblies of each of the two highly diverged mating types (MAT $\alpha$ and MATa) (Lengeler et al. 2002) and inferred phylogenies of each mating type separately (Fig. 6; Methods). The MAT $\alpha$ phylogeny largely matched the whole-genome phylogeny, with one major exception: VNBI $M A T \alpha$ appeared paraphyletic with respect to VNBII (Fig. 6A,B), with two distinct alleles-one comprised of some VNBI and the other clade comprised of the remaining VNBI plus all VNBII (bootstrap support: 100\%). These distinct clades did not map to specific subclades of VNBI in the whole-genome phylogeny but rather were intermixed.

The MATa phylogeny also differed from the whole-genome phylogeny (Fig. 6D,E) in that VNI and VNBI were closely related sister groups and separated by a large distance from VNBII (bootstrap support: $100 \%)$. Bootstrap support for the monophyly of VNBI with respect to VNI was limited (68\%), indicating the possibility that the VNI MATa allele was derived from a basal VNBI allele. Additionally, alleles cosegregating VNBI MATa isolates with VNI and VNBII MATa isolates extend $\sim 5.3$ and $3.5 \mathrm{~kb}$, respectively, upstream of the $5^{\prime}$ end of the MAT locus (Supplemental Fig. S8). This suggests a local introgression of the MATa locus from VNBI into VNI, consistent with previous reports of hotspots that flank the MAT region (Hsueh et al. 2006; Sun et al. 2012).

The MAT $\alpha$ and MATa alleles also showed distinct patterns of genetic diversity. Both alleles showed spikes of high genetic variation in a small number of hotspots (Fig. $6 \mathrm{C}, \mathrm{F}: \pi$ ); all of the regions corresponded to long terminal repeats in the annotations of the mating type loci, suggesting only limited variation has accumulated in functional genes. Within MAT $\alpha$, VNI alleles had significantly fewer singleton polymorphisms than either VNBI or VNBII, resulting in shorter terminal branch lengths (Wilcoxon rank-sum test, $P$ $<3.8 \times 10^{-5}$ and $P<5.2 \times 10^{-6}$, respectively) (Fig. 6B). Although this could be the result of mixing within VNI but not VNBI or VNBII, the same pattern was observed in the whole-genome data (Wilcoxon rank-sum test, $P<2.2 \times 10^{-16}$ and $P<1.7 \times 10^{-8}$ ) (Fig. 
A $\bar{s}$

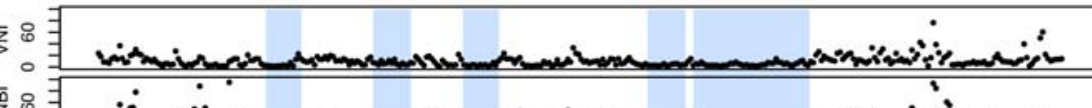

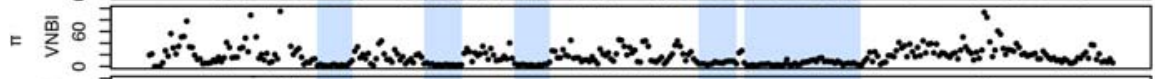

要过

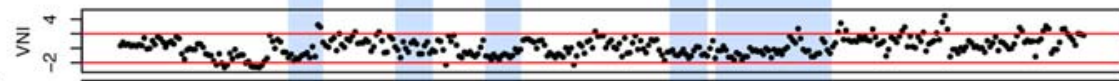
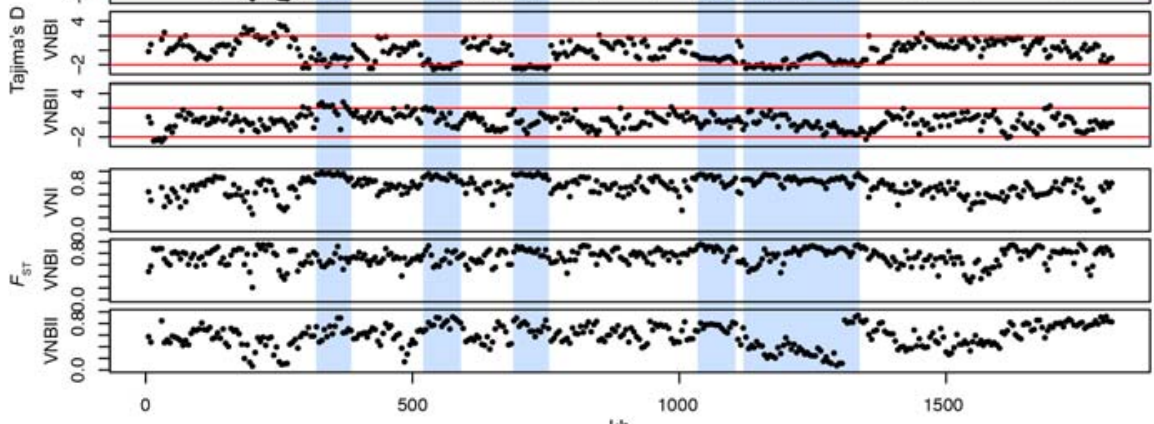

$\mathrm{kb}$
B
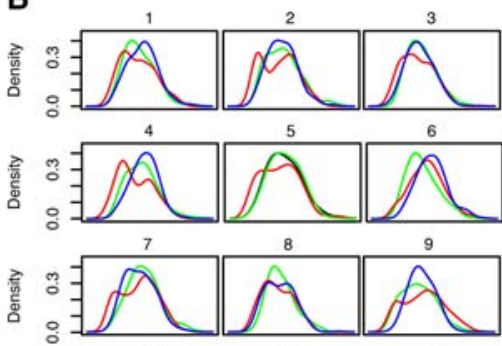

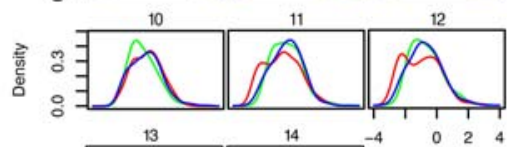

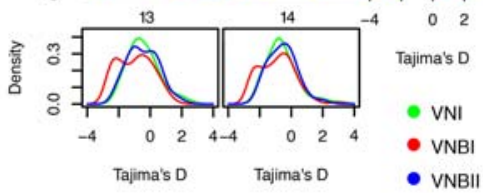

Figure 5. Population genomic analysis revealed long tracts of low genetic diversity and Tajima's $D$ in VNI and VNBI. (A) Low diversity tracts are shown here in Chromosome 5. In VNBI, these tracts are interspersed between regions of high diversity. Vertical blue bars highlight these areas of reduced diversity and Tajima's $D$ in VNBI, which are generally accompanied by high $F_{\mathrm{ST}}$ between populations. Statistics shown include $\pi$, Tajima's $D$, and $F_{\mathrm{ST}}$. $(B)$ Density distribution of Tajima's $D$ across all 14 chromosomes for VNI, VNBI, and VNBII. VNI and VNBIl show predominantly unimodal distributions for most chromosomes, and VNBI shows a bimodal distribution for all chromosomes except Chromosome 6.

1), suggesting an overall increase in genetic variation in VNBI or VNBII relative to VNI may be responsible for the difference. Between MAT $\alpha$ and MATa, MATa alleles had significantly more singleton polymorphisms than MAT $\alpha$, even when VNI isolates were excluded (Wilcoxon rank-sum test, $P<1.3 \times 10^{-6}$ ) (Fig. 6B, E), which could indicate less mixing between MATa alleles. Extensive linkage disequilibrium was seen in both MAT alleles $\left(\mathrm{R}^{2}\right)$ (Fig. 6C,F), suggesting that despite recombination occurring at the whole-genome level within VNI, VNBI, and VNBII, limited or no recombination was occurring within the mating type loci. In total, these analyses suggest that each mating type locus in $C$. neoformans has followed a distinct evolutionary trajectory from the remainder of the nuclear genome.

\section{Discussion}

Phylogenomic analysis presented here provided strong support for a deep split separating the VNB lineage into VNBI and VNBII, and further population genomic analysis revealed a lack of recombination between the two. These lineages also differed in their ability to be recovered from the environment, respond to oxidative stress, and melanize, and to a lesser extent the presence of both mating types (MATa was less common in VNBII). Based on these genomic and phenotypic differences, we propose that VNBI and VNBII should be considered two separate lineages. The lower frequency of isolates from environmental sources in VNBII could be due to an increased ability for VNBII isolates to colonize humans; there also may be geographic or niche differences in VNBII environmental populations. Despite the fact that melanization is used to identify isolates from the environment (i.e., light brown isolates are detected but pure white isolates are not), it is unlikely that decreased melanization of VNBII caused the group to be undersampled as most VNBII isolates melanized to at least some degree. Further genotypic and phenotypic evaluation will be re- quired to better delineate and understand the clinical ramifications of the differences between VNBI and VNBII.

Both "into" and "out of" Africa hypotheses have been proposed for VNI (Litvintseva et al. 2011). However, given the existence of the sub-Saharan Africa endemic VNIc and the presence of African isolates spread across VNIa and VNIb, the "into" hypothesis seems unlikely. More likely, the three VNI sublineages diversified and expanded within Africa and then two were globally disseminated at a later date, possibly by migration of birds and humans. Why VNI but not VNBI or VNBII would be globally dispersed is unclear. Although the data presented here support an African origin for VNB, a small number of South America VNB isolates have been described, including a previous analysis of nuclear loci that placed two of these isolates close to Bt22 (Ngamskulrungroj et al. 2009), identified here as a VNBI isolate. Comprehensive sequencing of South American VNB isolates will be required to confidently assess the origins of VNB diversity.

Given the lack of overlap between the tracts of low Tajima's $D$ and the regions identified as under selection, we hypothesize that the loss of diversity in VNI and VNBI are the result of previous demographic events, such as population bottlenecks, rather than selective sweeps fixing genes under selection. The global VNI lineage, with low overall diversity, likely emerged from a small founder population that may have undergone repeated bottlenecks while spreading geographically. Why the VNBI lineage, endemic to southeastern Africa, would have undergone a major demographic event, is unclear, as it appears to be the most well-represented lineage in current environmental sampling.

Previous analyses have highlighted the presence of recombination between isolates of different lineages based on MLST gene analysis (Litvintseva et al. 2006; Chen et al. 2015). The resolution possible with whole-genome data demonstrates that many of these isolates predicted to be recombinant by analysis of single genes such as SOD1 are predominantly a single ancestry based on whole-genome analyses (e.g., Bt65, Bt109). The detection of

\section{Genome Research}

www.genome.org 
Table 3. Inositol transport and utilization genes under selection in individual lineages

\begin{tabular}{lllll}
\hline H99 locus & Gene & VNI & VNBI & VNBII \\
\hline CNAG_00097 & Inositol transporter ITR1 & + & - & + \\
CNAG_04552 & Inositol transporter ITR1A & + & + & + \\
CNAG_00864 & Inositol transporter ITR2 & - & + & - \\
CNAG_05377 & Inositol transporter ITR3 & + & - & - \\
CNAG_00867 & Inositol transporter ITR3A & - & + & - \\
CNAG_05667 & Inositol transporter ITR3B & - & + & - \\
CNAG_05329 & myo-inositol 2-dehydrogenase & + & - & + \\
CNAG_05320 & Inositol 2-dehydrogenase & + & + & - \\
CNAG_05321 & Inositol 2-dehydrogenase & + & + & - \\
CNAG_06530 & Inositol 2-dehydrogenase & + & - & - \\
CNAG_05316 & Inositol oxygenase & + & - & - \\
\hline
\end{tabular}

Genes were present in the top 5\% of windows evaluated with the CLR test for each lineage.

small introgressions in VNBI, VNBII, and African VNI isolates suggests that a limited amount of recombination is occurring in Africa where the three groups overlap in distribution. Given the clinical significance of genes in these regions such as ERG11, better under- standing of the phylogenetic and geographic distribution of introgressions may be of clinical importance.

VNI showed a level of intragroup recombination comparable to VNBI and VNBII. This was unexpected as both mating types are common in the VNB lineages, suggesting sexual reproduction would dominate in $\mathrm{VNB}$, while the a mating type is rare in VNI, suggesting predominance of asexual reproduction in VNI. However, the high level of recombination seen in VNI may indicate unisexual mating in VNI is nearly as common as bisexual mating in VNBI and VNBII. It has been shown that unisexual mating between isolates of C. neoformans var. neoformans (serotype D) can occur and involves a complete meiotic cycle with recombination, similar to bisexual mating (Lin et al. 2005; Sun et al. 2014; Fu et al. 2015).

These population genomic analyses can also be used to evaluate lineage and species boundaries that are currently under debate for Cryptococcus (Hagen et al. 2015; Kwon-Chung et al. 2017). A multilocus viewpoint is critical for a comprehensive understanding of recombination and incomplete lineage sorting within a species, as recently shown for the asexual fungus Alternaria (Stewart et al. 2014). Despite high bootstrap support for the monophyly of the four population subdivisions (VNI, VNII, VNBI, and VNBII) in the whole-genome phylogenetic tree,
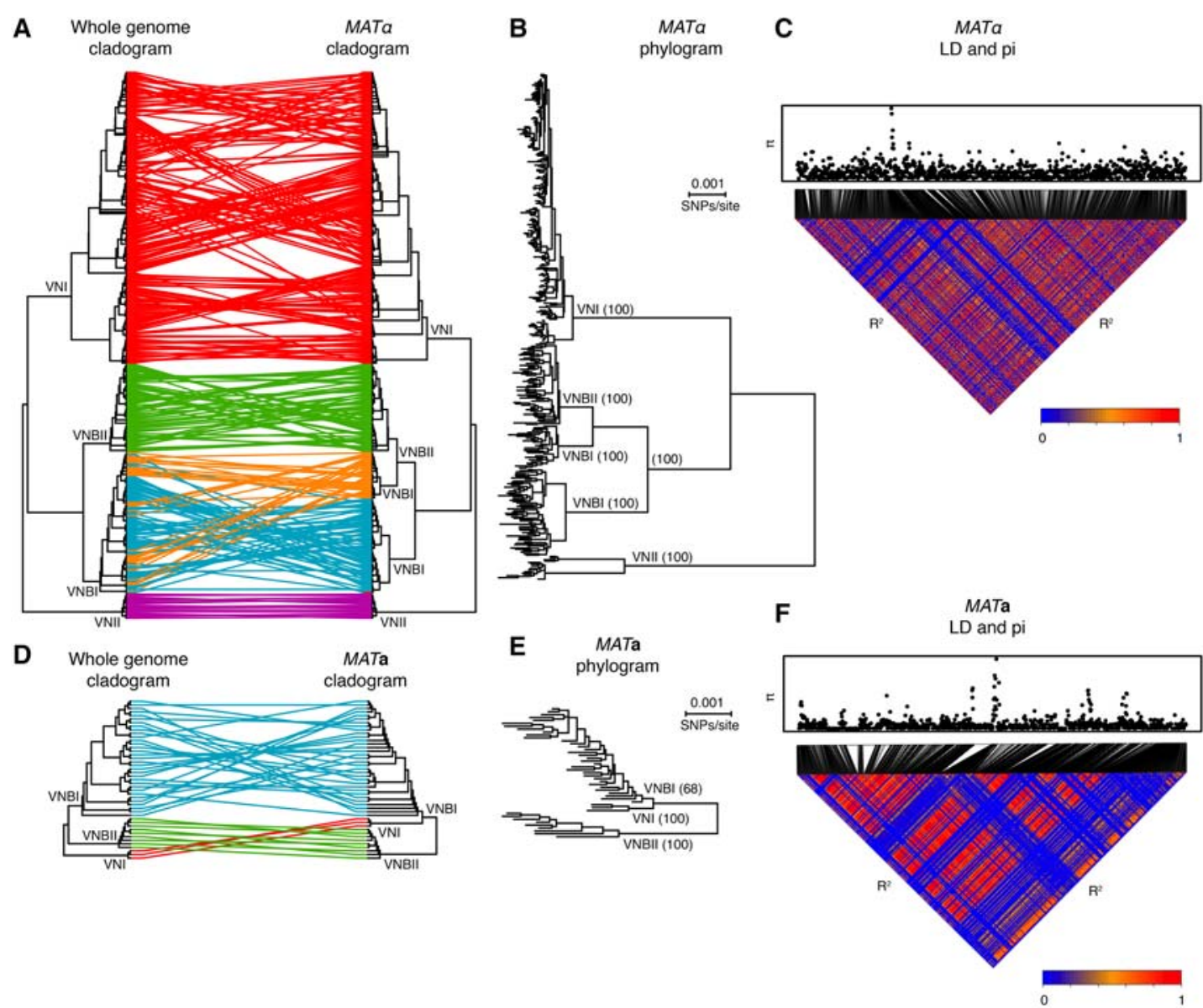

Figure 6. Phylogenetic and linkage analyses reveal distinct evolutionary trajectories of the MAT locus alleles. ( $A$ ) The topology of the whole-genome phylogeny and MAT $\alpha$ phylogeny are compared as cladograms. VNBI isolates contain two distinct $\alpha$ alleles. (B) MAT $\alpha$ phylogram showing branch lengths and bootstrap support for major clades. (C) Linkage disequilibrium $\left(R^{2}\right)$ and diversity $(\pi)$ across the MAT $\alpha$ locus. $(D)$ Topology of the whole-genome phylogeny and MATa phylogeny are compared as cladograms. (E) MATa phylogram showing branch lengths and bootstrap support for major clades. VNI is a close sister group to VNBI, in contrast to the whole-genome phylogeny, and there is limited support for monophyly of the VNI MATa allele with respect to VNBI (bootstrap support: 68\%). $(F)$ Linkage disequilibrium $\left(R^{2}\right)$ and diversity $(\pi)$ across the MATa locus. 
the detection of recombination between the lineages, including small introgressions, suggests that isolates from the different lineages mate and form recombinant progeny; further analysis including hybrid strains (Chen et al. 2015) will help clarify if these lineages represent varieties or sibling species. Although whole-genome analyses may largely recapitulate the phylogenetic relationships revealed from MLST studies, they can also reveal complex genetic exchange within a species at a level of detail not possible in smaller scale studies.

Phylogenetic analyses of the MAT alleles suggest that these alleles have followed distinct evolutionary trajectories from the remainder of the nuclear genome, involving incomplete sorting or genetic mixing following divergence of the groups, as seen in the paraphyly of the VNBI MAT $\alpha$ allele and close relationship of the VNI MATa allele to VNBI. These observations suggest an outline of the evolution of mating in these groups: the presence of both mating types is ancestral in VNBI and VNBII, and sexual reproduction in these groups involves opposite as well as potentially same-sex mating. However, VNI may have diverged with only the $M A T \alpha$ mating type, possibly due to a small founding population size. This would have provided evolutionary pressure for unisexual reproduction, as is suggested by the evidence of extensive recombination within the nuclear genomes of VNI isolates. Following the divergence of VNI from VNBI and VNBII, we speculate that VNI then reacquired the MATa allele from a VNBI-like ancestor.

The selection and association analyses, along with the phenotypic assays, provided clues to both selection pressures experienced by $C$. neoformans in the environment and which of these traits may translate to pathogenicity in humans. Selection on both inositol transport and utilization could affect the lineage-specific host range of $C$. neoformans and easily translate to altered virulence levels in humans, as inositol is abundant in the human brain (Fisher et al. 2002) and is required by C. neoformans for virulence (Shea et al. 2006) and mating (Xue et al. 2010). Gene expansions of inositol transporters in $C$. neoformans have been previously proposed to have evolved for growth on trees, yet preadapted $C$. neoformans for growth in the human brain (Xue et al. 2010; Xue 2012). Selection on xylose facilitators suggests potential adaptation to different tree hosts with different chemical compositions. GWAS analysis also revealed a gene known to be involved in the yeast-hyphal transition. As the inability to maintain yeast phase is known to correlate with decreased virulence (Wang et al. 2012), it is easy to hypothesize that natural adaptations to environments, where increased time in the yeast state would be selected for, could result in isolates with increased virulence in humans. Future genomic and phenotypic analyses focused on VNI may help better understand the specific properties that enabled the global dispersal of this lineage.

It was initially surprising that within VNBI, environmental isolates were more resistant to oxidative stress and more heavily melanized than clinical isolates, particularly given that melanization is a known virulence factor in humans. However, the diversity of stressors in the environment is likely greater than within the human host, and it has already been hypothesized that the ability of C. neoformans to survive in macrophages was derived from an ability to defend against predatory protozoa in the environment (Steenbergen et al. 2001). Further experiments are needed to disentangle the stresses faced in these two contexts and the differential ability of environmental and clinical isolates to respond to these stresses. Here, GWAS analysis linked loss-of-function mutations in the transcription factor $B Z P 4$ to significantly reduced melanization in a number of unrelated clinical isolates, highlighting the po- tential clinical impact of this complex interplay. In sum, these data provide insight into how selection pressure over the evolutionary history of $C$. neoformans may have preadapted this species to successful invasion of the human nervous system and suggests many pathways to explore for differential virulence within standing genetic diversity or outbreaks.

\section{Methods}

\section{Sample selection}

We selected 387 isolates for analysis: 185 from VNI, 186 from VNB, and 16 from VNII. We sought to represent isolation sources and mating types as evenly as possible for each of VNI and VNB, and we therefore preferentially chose isolates containing MATa and those isolated from environmental sources, as both are less frequently collected, particularly for VNI. We also included a limited number of VNII isolates, but did not focus on this lineage as it is rarely isolated. The majority of isolates, particularly VNB isolates, were collected in Botswana, and more specific collection details of these isolates are given in Supplemental Methods and a previous MLST analysis (Chen et al. 2015).

\section{Sample preparation, sequencing, and variant identification}

Samples were prepared for DNA extraction as described (Supplemental Methods). Genomic DNA was sheared to $\sim 250 \mathrm{bp}$ using a Covaris LE instrument and adapted for Illumina sequencing as previously described (Fisher et al. 2011). Libraries were sequenced on an Illumina HiSeq to generate 101 base reads. Reads were aligned to the C. neoformans var. grubii $\mathrm{H} 99$ assembly (GenBank accession GCA_000149245.2) using BWA-MEM version 0.7.12 (Li 2013). Variants were then identified using GATK version 3.4 (McKenna et al. 2010) as described (Supplemental Methods) and functionally annotated with SnpEff version 4.2 (Cingolani et al. 2012). Read data from 46 previously sequenced Zambian isolates (Vanhove et al. 2017) were downloaded from NCBI (BioProject PRJEB13814), and variants were called for all samples as described above.

\section{Phylogenetic analysis}

For phylogenetic analysis, the 1,069,080 sites with an unambiguous SNP in at least one isolate and with ambiguity in at most $10 \%$ of isolates were concatenated; insertions or deletions at these sites were treated as ambiguous to maintain the alignment. Phylogenetic trees were estimated using RAxML version 8.2.4 (Stamatakis 2014) under the GTRCAT model in rapid bootstrapping mode. We then placed the 46 previously sequenced Zambian isolates (Vanhove et al. 2017) in phylogenetic context with the isolates in this study using FastTree version 2.1.8 compiled for double precision (Price et al. 2010).

\section{Population genomic analysis}

Population structure was investigated using multiple approaches. Principal components analysis was run on all variants using SMARTPCA (Patterson et al. 2006). Major ancestry subdivisions were delineated using STRUCTURE (Pritchard et al. 2000) v2.3.4 in the site-by-site mode and $k=4$, with $15 \%$ of the positions randomly subsampled from those containing at least two variants and missing in $<5 \%$ of isolates. Identification of VNI subdivisions and smaller interlineage introgressions using STRUCTURE were performed as described (Supplemental Methods). Population genomic metrics were calculated using VCFtools version 1.14 (Danecek et al. 2011) and PopGenome (Pfeifer et al. 2014); further

\section{Genome Research}

www.genome.org 
details are given in Supplemental Methods. The CLR selection metric was also calculated using PopGenome, and enrichment tests were conducted using Fisher's exact test corrected with the Benjamini-Hochberg method for multiple comparisons (Supplemental Methods) (Benjamini and Hochberg 1995).

\section{Association analysis}

For each phenotype, two matrices of these features were constructed from the variant calls of all isolates. In the first matrix, rare variants (those at $\leq 5 \%$ frequency) were collapsed by gene or intergenic region, and common variants (those at $>5 \%$ frequency) were considered independently. In the second matrix, we assessed whether each gene had a loss-of-function mutation (defined as a "HIGH" impact mutation by SnpEff), regardless of the frequency of the mutation. Each variant matrix was then analyzed with GEMMA (Zhou and Stephens 2012) using a univariate linear mixed model and a relatedness matrix to account for population stratification. Score test results of both analyses were then combined and examined. ERG11 and AFR1 copy number of drug resistant isolates were estimated as the normalized read depth of these genes from whole-genome BWA-MEM alignments, where the per base depth calculated using SAMtools (Li et al. 2009) v1.3 mpileup was summed across each gene and normalized to the average genome depth.

\section{Phenotypic assays}

Freezer stocks were grown, plated, and pinned in 1536 array format as described (Supplemental Methods); each single colony selected from the grown freezer stock became a $4 \times 4$ block of 16 colonies in two 1536 arrays. Four conditions were assayed: $5.6 \mathrm{mM} \mathrm{H}_{2} \mathrm{O}_{2}, 1.0$ $\mathrm{mM}$ paraquat, $10 \mu \mathrm{g} / \mathrm{mL}$ fluconazole, and $0.1 \mathrm{mg} / \mathrm{mL} \mathrm{L}-\mathrm{DOPA}$. Arrays were incubated at $30^{\circ} \mathrm{C}$ and images were acquired 1-, 2-, and 3-d post-pinning. Day 2 images were analyzed using gitter (Wagih and Parts 2014) to assess either colony size in the case of $\mathrm{H}_{2} \mathrm{O}_{2}$, paraquat, or fluconazole, or brightness in the case of LDOPA. Each condition was tested in either one $\left(\mathrm{H}_{2} \mathrm{O}_{2}\right)$, two (LDOPA and fluconazole), or three (paraquat) independent experiments. All independent experiments replicated well $\left(R^{2}, \mathrm{~L}-\mathrm{DOPA}\right.$ $=0.51$, fluconazole $=0.59$, paraquat $=0.47-0.63)$. We then identified differences between VNBI and VNBII clinical isolates, and VNBI environmental and clinical isolates, using the MannWhitney $U$ test. As $C$. neoformans is identified in the environment (but not in the clinic) by the ability to melanize to some degree (i.e., light brown isolates are detected but pure white isolates are not), in the comparison of melanization of environmental and clinical VNBI isolates, we excluded isolates that were unable to melanize (brightness $\geq 0.6$ ), because the sampling of those isolates would be biased toward clinical samples.

\section{Mating type analysis}

Analyses of the mating type loci were done with the same methodology as the whole-genome analyses with the following two exceptions. First, reads were mapped to the appropriate high quality locus-specific reference, either the H99 MAT $\alpha$ allele (GenBank accession AF542529) or the 125.91 MATa allele (GenBank accession AF542528). Second, linkage disequilibrium was calculated on the site level rather than in windows and visualized using the R package LDheatmap (Shin et al. 2006).

\section{Data Access}

All sequence data from this study have been submitted to NCBI BioProject (https://www.ncbi.nlm.nih.gov/bioproject) under ac- cession number PRJNA382844. Individual data set accession numbers are listed in Supplemental Table S1.

\section{Acknowledgments}

We thank Jose Munoz and three anonymous reviewers for providing helpful comments on the manuscript. This project has been funded in whole or in part with federal funds from the National Institute of Allergy and Infectious Diseases, National Institutes of Health, Department of Health and Human Services, under Grant Number U19AI110818 to the Broad Institute. Support to J.R.P. came from US Public Health Service Grants AI73896, AI93257. Support to S.S. and J.H. was from NIH/NIAID R37 AI39115-20 and RO1 AI50113-13. The use of product names in this manuscript does not imply their endorsement by the US Department of Health and Human Services. The findings and conclusions in this article are those of the authors and do not necessarily represent the views of the CDC. The clinical isolates were approved under Pro00029982 from the Duke Institutional Review Board in the United States and the Review Boards of Nyangabgwe and Princess Marina, Hospitals of Botswana.

Author contributions: C.A.C., A.P.L., and J.R.P. conceived and designed the project. M.R.H. provided the clinical isolates. C.G., T.Y., Y.C., and A.M.J. performed the laboratory experiments. C.A.D., S.M.S., C.-H.Y., C.A.C., S.S., J.H., and C.G. analyzed the data. C.A.D., C.A.C., and A.P.L. wrote the paper. C.A.C., J.R.P., and J.L.T. supervised and coordinated the project.

\section{References}

Benjamini Y, Hochberg Y. 1995. Controlling the false discovery rate: a practical and powerful approach to multiple testing. $J R$ Stat Soc Ser B Methodol 57: 289-300.

Brown SP, Cornforth DM, Mideo N. 2012. Evolution of virulence in opportunistic pathogens: generalism, plasticity, and control. Trends Microbiol 20: $336-342$.

Brown JC, Nelson J, VanderSluis B, Deshpande R, Butts A, Kagan S, Polacheck I, Krysan DJ, Myers CL, Madhani HD. 2014. Unraveling the biology of a fungal meningitis pathogen using chemical genetics. Cell 159: $1168-1187$.

Bulmer GS, Sans MD, Gunn CM. 1967. Cryptococcus neoformans I. Nonencapsulated mutants. J Bacteriol 94: 1475-1479.

Chacko N, Zhao Y, Yang E, Wang L, Cai JJ, Lin X. 2015. The lncRNA RZE1 controls cryptococcal morphological transition. PLoS Genet 11: e1005692.

Chayakulkeeree M, Perfect JR. 2006. Cryptococcosis. Infect Dis Clin North Am 20: 507-544, v-vi.

Chen Y, Litvintseva AP, Frazzitta AE, Haverkamp MR, Wang L, Fang C, Muthoga C, Mitchell TG, Perfect JR. 2015. Comparative analyses of clinical and environmental populations of Cryptococcus neoformans in Botswana. Mol Ecol 24: 3559-3571.

Chow EWL, Morrow CA, Djordjevic JT, Wood IA, Fraser JA. 2012. Microevolution of Cryptococcus neoformans driven by massive tandem gene amplification. Mol Biol Evol 29: 1987-2000.

Cingolani P, Platts A, Wang LL, Coon M, Nguyen T, Wang L, Land SJ, Lu X, Ruden DM. 2012. A program for annotating and predicting the effects of single nucleotide polymorphisms, SnpEff: SNPs in the genome of Drosophila melanogaster strain $w^{1118}$; iso-2; iso-3. Fly (Austin) 6: 80-92.

Cox GM, Mukherjee J, Cole GT, Casadevall A, Perfect JR. 2000. Urease as a virulence factor in experimental cryptococcosis. Infect Immun 68: 443-448.

Cox GM, McDade HC, Chen SCA, Tucker SC, Gottfredsson M, Wright LC, Sorrell TC, Leidich SD, Casadevall A, Ghannoum MA, et al. 2001. Extracellular phospholipase activity is a virulence factor for Cryptococcus neoformans. Mol Microbiol 39: 166-175.

Danecek P, Auton A, Abecasis G, Albers CA, Banks E, DePristo MA Handsaker RE, Lunter G, Marth GT, Sherry ST, et al. 2011. The variant call format and VCFtools. Bioinformatics 27: 2156-2158.

de Jesús-Berríos M, Liu L, Nussbaum JC, Cox GM, Stamler JS, Heitman J. 2003. Enzymes that counteract nitrosative stress promote fungal virulence. Curr Biol 13: 1963-1968.

D'Souza CA, Kronstad JW, Taylor G, Warren R, Yuen M, Hu G, Jung WH, Sham A, Kidd SE, Tangen K, et al. 2011. Genome variation in 
Cryptococcus gattii, an emerging pathogen of immunocompetent hosts. mBio 2: e00342-10.

Farrer RA, Desjardins CA, Sakthikumar S, Gujja S, Saif S, Zeng Q, Chen Y, Voelz K, Heitman J, May RC, et al. 2015. Genome evolution and innovation across the four major lineages of Cryptococcus gattii. mBio 6: e00868-15.

Ferreira-Paim K, Andrade-Silva L, Fonseca FM, Ferreira TB, Mora DJ, Andrade-Silva J, Khan A, Dao A, Reis EC, Almeida MTG, et al. 2017. MLST-based population genetic analysis in a global context reveals clonality amongst Cryptococcus neoformans var. grubii VNI isolates from HIV patients in Southeastern Brazil. PLoS Negl Trop Dis 11: e0005223.

Fisher SK, Novak JE, Agranoff BW. 2002. Inositol and higher inositol phosphates in neural tissues: homeostasis, metabolism and functional significance. J Neurochem 82: 736-754.

Fisher S, Barry A, Abreu J, Minie B, Nolan J, Delorey TM, Young G, Fennell TJ, Allen A, Ambrogio L, et al. 2011. A scalable, fully automated process for construction of sequence-ready human exome targeted capture libraries. Genome Biol 12: R1.

Fromtling RA, Abruzzo GK, Ruiz A. 1989. Virulence and antifungal susceptibility of environmental and clinical isolates of Cryptococcus neoformans from Puerto Rico. Mycopathologia 106: 163-166.

Fu C, Sun S, Billmyre RB, Roach KC, Heitman J. 2015. Unisexual versus bisexual mating in Cryptococcus neoformans: consequences and biological impacts. Fungal Genet Biol 78: 65-75.

Geddes JMH, Croll D, Caza M, Stoynov N, Foster LJ, Kronstad JW. 2015 Secretome profiling of Cryptococcus neoformans reveals regulation of a subset of virulence-associated proteins and potential biomarkers by protein kinase A. BMC Microbiol 15: 206.

Hagen F, Khayhan K, Theelen B, Kolecka A, Polacheck I, Sionov E, Falk R, Parnmen S, Lumbsch HT, Boekhout T. 2015. Recognition of seven species in the Cryptococcus gattii/Cryptococcus neoformans species complex. Fungal Genet Biol 78: 16-48.

Hiremath SS, Chowdhary A, Kowshik T, Randhawa HS, Sun S, Xu J. 2008. Long-distance dispersal and recombination in environmental populations of Cryptococcus neoformans var. grubii from India. Microbiology 154: $1513-1524$.

Hsueh YP, Idnurm A, Heitman J. 2006. Recombination hotspots flank the Cryptococcus mating-type locus: implications for the evolution of a fungal sex chromosome. PLoS Genet 2: e184.

Janbon G, Ormerod KL, Paulet D, Byrnes EJ III, Yadav V, Chatterjee G, Mullapudi N, Hon CC, Billmyre RB, Brunel F, et al. 2014. Analysis of the genome and transcriptome of Cryptococcus neoformans var. grubii reveals complex RNA expression and microevolution leading to virulence attenuation. PLoS Genet 10: e1004261.

Jung KW, Yang DH, Maeng S, Lee KT, So YS, Hong J, Choi J, Byun HJ, Kim H, Bang S, et al. 2015. Systematic functional profiling of transcription factor networks in Cryptococcus neoformans. Nat Commun 6: 6757.

Kmetzsch L, Staats CC, Simon E, Fonseca FL, de Oliveira DL, Sobrino L, Rodrigues J, Leal AL, Nimrichter L, Rodrigues ML, et al. 2010. The vacuolar $\mathrm{Ca}^{2+}$ exchanger Vcx1 Is involved in calcineurin-dependent $\mathrm{Ca}^{2+}$ tolerance and virulence in Cryptococcus neoformans. Eukaryot Cell 9: 1798-1805.

Kozubowski L, Lee SC, Heitman J. 2009. Signalling pathways in the pathogenesis of Cryptococcus. Cell Microbiol 11: $370-380$

Kwon-Chung KJ, Rhodes JC. 1986. Encapsulation and melanin formation as indicators of virulence in Cryptococcus neoformans. Infect Immun 51: 218-223.

Kwon-Chung KJ, Bennett JE, Wickes BL, Meyer W, Cuomo CA, Wollenburg KR, Bicanic TA, Castañeda E, Chang YC, Chen J, et al. 2017. The case for adopting the "species complex" nomenclature for the etiologic agents of Cryptococcosis. mSphere 2: e00357-16.

Lengeler KB, Fox DS, Fraser JA, Allen A, Forrester K, Dietrich FS, Heitman J. 2002. Mating-type locus of Cryptococcus neoformans: a step in the evolution of sex chromosomes. Eukaryot Cell 1: 704-718.

Li H. 2013. Aligning sequence reads, clone sequences and assembly contigs with BWA-MEM. arXiv:o1303.3997.

Li H, Handsaker B, Wysoker A, Fennell T, Ruan J, Homer N, Marth G, Abecasis G, Durbin R; 1000 Genome Project Data Processing Subgroup. 2009. The Sequence Alignment/Map format and SAMtools. Bioinformatics 25: 2078-2079.

Lin X, Hull CM, Heitman J. 2005. Sexual reproduction between partners of the same mating type in Cryptococcus neoformans. Nature 434: 1017-1021.

Liti G, Carter DM, Moses AM, Warringer J, Parts L, James SA, Davey RP, Roberts IN, Burt A, Koufopanou V, et al. 2009. Population genomics of domestic and wild yeasts. Nature 458: 337-341.

Litvintseva AP, Mitchell TG. 2009. Most environmental isolates of Cryptococcus neoformans var. grubii (serotype A) are not lethal for mice. Infect Immun 77: 3188-3195.
Litvintseva AP, Marra RE, Nielsen K, Heitman J, Vilgalys R, Mitchell TG. 2003. Evidence of sexual recombination among Cryptococcus neoforman serotype A isolates in sub-Saharan Africa. Eukaryot Cell 2: 1162-1168.

Litvintseva AP, Thakur R, Vilgalys R, Mitchell TG. 2006. Multilocus sequence typing reveals three genetic subpopulations of Cryptococcus neoformans var. grubii (serotype A), including a unique population in Botswana. Genetics 172: 2223-2238.

Litvintseva AP, Carbone I, Rossouw J, Thakur R, Govender NP, Mitchell TG. 2011. Evidence that the human pathogenic fungus Cryptococcus neoformans var. grubii may have evolved in Africa. PLoS One 6: e19688.

Liu TB, Xue C. 2014. Fbp1-mediated ubiquitin-proteasome pathway controls Cryptococcus neoformans virulence by regulating fungal intracellular growth in macrophages. Infect Immun 82: 557-568.

Liu OW, Chun CD, Chow ED, Chen C, Madhani HD, Noble SM. 2008. Systematic genetic analysis of virulence in the human fungal pathogen Cryptococcus neoformans. Cell 135: 174-188.

Loftus BJ, Fung E, Roncaglia P, Rowley D, Amedeo P, Bruno D, Vamathevan J, Miranda M, Anderson IJ, Fraser JA, et al. 2005. The genome of the basidiomycetous yeast and human pathogen Cryptococcus neoformans. Science 307: 1321-1324.

McKenna A, Hanna M, Banks E, Sivachenko A, Cibulskis K, Kernytsky A, Garimella K, Altshuler D, Gabriel S, Daly M, et al. 2010. The Genome Analysis Toolkit: a MapReduce framework for analyzing next-generation DNA sequencing data. Genome Res 20: 1297-1303.

Morrow CA, Lee IR, Chow EW, Ormerod KL, Goldinger A, Byrnes EJ, Nielsen K, Heitman J, Schirra HJ, Fraser JA. 2012. A unique chromosomal rearrangement in the Cryptococcus neoformans var. grubii type strain enhances key phenotypes associated with virulence. mBio 3: e00310-11.

Ngamskulrungroj P, Gilgado F, Faganello J, Litvintseva AP, Leal AL, Tsui KM, Mitchell TG, Vainstein MH, Meyer W. 2009. Genetic diversity of the Cryptococcus species complex suggests that Cryptococcus gattii deserves to have varieties. PLoS One 4: e5862.

Nielsen R, Williamson S, Kim Y, Hubisz MJ, Clark AG, Bustamante C. 2005 Genomic scans for selective sweeps using SNP data. Genome Res 15: $1566-1575$.

O'Meara TR, Alspaugh JA. 2012. The Cryptococcus neoformans capsule: a sword and a shield. Clin Microbiol Rev 25: 387-408.

Park BJ, Wannemuehler KA, Marston BJ, Govender N, Pappas PG, Chiller TM. 2009. Estimation of the current global burden of cryptococcal meningitis among persons living with HIV/AIDS. AIDS Lond Engl 23: $525-530$.

Patterson N, Price AL, Reich D. 2006. Population structure and eigenanalysis. PLoS Genet 2: e190.

Pfeifer B, Wittelsbürger U, Ramos-Onsins SE, Lercher MJ. 2014 PopGenome: an efficient Swiss army knife for population genomic analyses in R. Mol Biol Evol 31: 1929-1936.

Price MN, Dehal PS, Arkin AP. 2010. FastTree 2 - approximately maximumlikelihood trees for large alignments. PLoS One 5: e9490.

Pritchard JK, Stephens M, Donnelly P. 2000. Inference of population structure using multilocus genotype data. Genetics 155: 945-959.

Salas SD, Bennett JE, Kwon-Chung KJ, Perfect JR, Williamson PR. 1996. Effect of the laccase gene CNLAC1, on virulence of Cryptococcus neoformans. J Exp Med 184: 377-386.

Shea JM, Henry JL, Poeta MD. 2006. Lipid metabolism in Cryptococcus neoformans. FEMS Yeast Res 6: 469-479.

Shin JH, Blay S, McNeney B, Graham J. 2006. LDheatmap: an R function for graphical display of pairwise linkage disequilibria between single nucleotide polymorphisms. J Stat Softw 16. doi: 10.18637/jss.v016.c03.

Specht CA, Lee CK, Huang H, Tipper DJ, Shen ZT, Lodge JK, Leszyk J, Ostroff GR, Levitz SM. 2015. Protection against experimental cryptococcosis following vaccination with glucan particles containing Cryptococcus alkaline extracts. mBio 6: e01905-15.

Stamatakis A. 2014. RAxML version 8: a tool for phylogenetic analysis and post-analysis of large phylogenies. Bioinformatics 30: 1312-1313.

Steenbergen JN, Shuman HA, Casadevall A. 2001. Cryptococcus neoformans interactions with amoebae suggest an explanation for its virulence and intracellular pathogenic strategy in macrophages. Proc Natl Acad Sci 98: 15245-15250.

Stewart JE, Timmer LW, Lawrence CB, Pryor BM, Peever TL. 2014. Discord between morphological and phylogenetic species boundaries: incomplete lineage sorting and recombination results in fuzzy species boundaries in an asexual fungal pathogen. BMC Evol Biol 14: 38.

Sun S, Hsueh YP, Heitman J. 2012. Gene conversion occurs within the mating-type locus of Cryptococcus neoformans during sexual reproduction. PLoS Genet 8: e1002810.

Sun S, Billmyre RB, Mieczkowski PA, Heitman J. 2014. Unisexual reproduction drives meiotic recombination and phenotypic and karyotypic plasticity in Cryptococcus neoformans. PLoS Genet 10: e1004849.

Tajima F. 1989. The effect of change in population size on DNA polymorphism. Genetics 123: 597-601.

\section{Genome Research}

www.genome.org 
Upadhya R, Campbell LT, Donlin MJ, Aurora R, Lodge JK. 2013. Global transcriptome profile of Cryptococcus neoformans during exposure to hydrogen peroxide induced oxidative stress. PLoS One 8: e55110.

Vanhove M, Beale MA, Rhodes J, Chanda D, Lakhi S, Kwenda G, Molloy S, Karunaharan N, Stone N, Harrison TS, et al. 2017. Genomic epidemiology of Cryptococcus yeasts identifies adaptation to environmental niches underpinning infection across an African HIV/AIDS cohort. Mol Ecol 26: 1991-2005.

Wagih O, Parts L. 2014. gitter: a robust and accurate method for quantification of colony sizes from plate images. G3 (Bethesda) 4: 547-552.

Wang Y, Liu TB, Patel S, Jiang L, Xue C. 2011. The casein kinase I protein Cck1 regulates multiple signaling pathways and is essential for cell integrity and fungal virulence in Cryptococcus neoformans. Eukaryot Cell 10: $1455-1464$.
Wang L, Zhai B, Lin X. 2012. The link between morphotype transition and virulence in Cryptococcus neoformans. PLoS Pathog 8: e1002765.

Xue C. 2012. Cryptococcus and beyond-inositol utilization and its implications for the emergence of fungal virulence. PLoS Pathog 8: e1002869.

Xue C, Liu T, Chen L, Li W, Liu I, Kronstad JW, Seyfang A, Heitman J. 2010. Role of an expanded inositol transporter repertoire in Cryptococcus neoformans sexual reproduction and virulence. mBio 1: e00084-10.

Zhou X, Stephens M. 2012. Genome-wide efficient mixed-model analysis for association studies. Nat Genet 44: 821-824.

Received November 22, 2016; accepted in revised form May 1, 2017. 


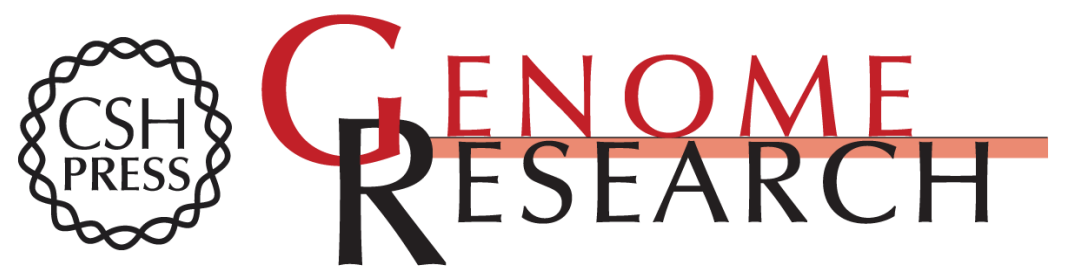

\section{Population genomics and the evolution of virulence in the fungal pathogen Cryptococcus neoformans}

Christopher A. Desjardins, Charles Giamberardino, Sean M. Sykes, et al.

Genome Res. 2017 27: 1207-1219 originally published online June 13, 2017 Access the most recent version at doi:10.1101/gr.218727.116

Supplemental Material

\section{References}

Open Access

Creative Commons

License

Email Alerting Service
http://genome.cshlp.org/content/suppl/2017/06/05/gr.218727.116.DC1

This article cites 69 articles, 24 of which can be accessed free at: http://genome.cshlp.org/content/27/7/1207.full.html\#ref-list-1

Freely available online through the Genome Research Open Access option.

This article, published in Genome Research, is available under a Creative Commons License (Attribution 4.0 International), as described at http://creativecommons.org/licenses/by/4.0/.

Receive free email alerts when new articles cite this article - sign up in the box at the top right corner of the article or click here.

\section{Affordable, Accurate Sequencing.}

